\title{
Research Article \\ Partial Finite-Time Synchronization of Switched Stochastic Chua's Circuits via Sliding-Mode Control
}

\author{
Zhang-Lin Wan, ${ }^{1}$ Yi-You Hou, ${ }^{2}$ Teh-Lu Liao, ${ }^{1}$ and Jun-Juh Yan $^{3}$ \\ ${ }^{1}$ Department of Engineering Science, National Cheng Kung University, Tainan 701, Taiwan \\ ${ }^{2}$ Department of Electrical Engineering, Far East University, Tainan 744, Taiwan \\ ${ }^{3}$ Department of Computer and Communication, Shu-Te University, Kaohsiung 824, Taiwan \\ Correspondence should be addressed to Jun-Juh Yan, jjyan@mail.stu.edu.tw
}

Received 17 May 2011; Revised 21 September 2011; Accepted 22 September 2011

Academic Editor: Kwok W. Wong

Copyright (c) 2011 Zhang-Lin Wan et al. This is an open access article distributed under the Creative Commons Attribution License, which permits unrestricted use, distribution, and reproduction in any medium, provided the original work is properly cited.

This paper considers the problem of partial finite-time synchronization between switched stochastic Chua's circuits accompanied by a time-driven switching law. Based on the Ito formula and Lyapunov stability theory, a sliding-mode controller is developed to guarantee the synchronization of switched stochastic master-slave Chua's circuits and for the mean of error states to obtain the partial finite-time stability. Numerical simulations demonstrate the effectiveness of the proposed methods.

\section{Introduction}

The concept of chaos synchronization in message transmission has been extensively studied. The synchronization of chaos is a key technology in generating identical chaotic waveforms in the transmitter and receiver for signal decoding. Under the assumption that the structure of nonlinearity or matching condition is known, studies on chaos synchronization have been concerned with control methods and applications [1-5]. Many natural physical systems such as chemical processes, mechanical systems, and a variety of power systems can be described by hybrid models comprising continuous and discrete dynamic behaviors. A special case is a hybrid system composed of many subsystems and a rule that governs the switching between these subsystems. By neglecting the details of the discrete behavior and instead of considering all possible switching patterns for a certain class, a switched system may be derived from a hybrid system [6]. Recently, the problems of stability analysis and synchronization of switched systems have attracted a lot of attention [7-11]. In the present study, special chaotic systems whose gain is changed by switching rules are designed to force the speed of system response to be fast or slow, as with frequency modulation. 
Stochastic processes such as electrocardiography, stock market, and Brownian motion have been extensively investigated. Brownian motion is usually described by the Wiener process, which is a continuous-time stochastic process, and taken as the nature uncertainties and perturbations. The stability of stochastic systems has been extensively studied because the uncertainties and perturbations are similar to those in real-world systems [12-15]. Stochastic stability can be guaranteed by the Ito formula, which is derived by using Taylor series expansion. Recently, studies on stochastic chaos synchronization have received a lot of attention. As a special problem of chaotic systems, some kinds of control methods and convergence judgments have been conferred in this decade [16-19].

Sliding-mode control (SMC) is a special case of variable structure systems. By designing a switching surface and using a discontinuous control law, the trajectories of dynamic systems can be forced to slide along the fixed sliding manifold. Then, the system can be compelled to satisfy the desired performance. Generally speaking, the two main advantages of SMC are the uncomplicated dynamic behaviors of the system with the designed switching functions and strong robustness to system uncertainties. Many studies have been conducted on SMC [20-24]. The asymptotic stability theorem with the fundamental theory of Lyapunov has been used to prove the stability of systems. However, the stability over the finite horizon of time cannot be guaranteed. In many applications, it is desirable for the trajectories of the system to converge to a stable equilibrium state in finite time rather than asymptotically. Recently, a particular property of asymptotic stability, finite-time stability, has attracted research interest due to its feasibility and advantages. Based on this property, stability can be achieved within the settling time for many control methods [25-28]. However, few studies have focused on the finite-time stability of stochastic processes. Therefore, the present study designs a sliding-mode control scheme to force the error states of two switched stochastic Chua's circuits to converge to the sliding surface and for their mean value to converge to zero. In this paper, the partial finite-time stability means that there must be at least one state that can achieve finite-time stability, and other states can obtain asymptotical stability [29]. In order to ensure partial finite-time stability, the Ito formula and Lyapunov stability theory are used to guarantee the synchronization and the mean of the error states reaching zero for the switched stochastic Chua's circuits.

The rest of this paper is organized as follows. In Section 2, an appropriate switching surface and a sliding-mode controller are designed to drive the system trajectory to reach the sliding surface and stochastically synchronize the master-slave switched stochastic Chua's circuits on the sliding manifold. A numerical example is given in Section 3. Finally, conclusion is presented in Section 4. Note that throughout the remainder of this paper, the notation $E[x(t)]$ denotes the mean of $x(t) \cdot|x(t)|$ denotes the modulus of $x(t) \cdot \operatorname{sgn}(x(t))$ is defined as if $x(t)>0, \operatorname{sgn}(x(t))=1$; if $x(t)=0, \operatorname{sgn}(x(t))=0$; if $x(t)<0, \operatorname{sgn}(x(t))=-1$.

\section{System Description and Main Results}

Consider the Chua's circuit described as follows:

$$
\begin{gathered}
\dot{x}(t)=p(y(t)-x(t)-f(x(t))), \\
\dot{y}(t)=x(t)-y(t)+z(t), \\
\dot{z}(t)=-q y(t)-r z(t),
\end{gathered}
$$


where $x(t), y(t)$, and $z(t)$ are system states; $f(x(t))$ is a three-segment piecewise linear function $f(x(t))=b x(t)+(1 / 2)(a-b)[|x(t)+1|-|x(t)-1|]$ that satisfies the Lipschitz condition with Lipschitz constant $\ell>0 . a<-1,-1<b<0, p>0, q>0$, and $r>0$ are system parameters.

A set of nonlinear stochastic Chua's circuits are derived with the separate switching rules of the switched system. Master and slave stochastic switched systems are respectively described as follows:

$$
\begin{gathered}
d x_{m}(t)=\kappa_{\delta(t)}\left\{\left[p\left(y_{m}(t)-x_{m}(t)-f\left(x_{m}(t)\right)\right)\right] d t\right\}, \\
d y_{m}(t)=\kappa_{\delta(t)}\left\{\left[x_{m}(t)-y_{m}(t)+z_{m}(t)\right] d t\right\} \\
d z_{m}(t)=\kappa_{\delta(t)}\left\{\left[-q y_{m}(t)-r z_{m}(t)\right] d t\right\}, \\
d x_{S}(t)=\kappa_{\delta(t)}\left\{\left[p\left(y_{s}(t)-x_{s}(t)-f\left(x_{s}(t)\right)\right)\right] d t+\sigma_{1}(t) e_{x}(t) d w_{1}(t)\right\}, \\
d y_{s}(t)=\kappa_{\delta(t)}\left\{\left[x_{s}(t)-y_{s}(t)+z_{S}(t)-u(t)\right] d t+\sigma_{2}(t) e_{y}(t) d w_{2}(t)\right\}, \\
d z_{s}(t)=\kappa_{\delta(t)}\left\{\left[-q y_{s}(t)-r z_{s}(t)\right] d t+\sigma_{3}(t) e_{z}(t) d w_{3}(t)\right\},
\end{gathered}
$$

where $f\left(x_{m}(t)\right)=b x_{m}(t)+(1 / 2)(a-b)\left[\left|x_{m}(t)+1\right|-\left|x_{m}(t)-1\right|\right]$ and $f\left(x_{s}(t)\right)=b x_{s}(t)+(1 / 2)(a-$ b) $\left[\left|x_{s}(t)+1\right|-\left|x_{s}(t)-1\right|\right], u(t)$ is the sliding-mode control input, $\kappa_{\delta(t)} \geq 1$ is a time-driven switching gain, and $\delta(\cdot):[0, \infty) \rightarrow\{1,2, \ldots, N\}$ is a piecewise switching signal. Moreover, $\delta(t)=i$ implies that the $i$ th switching gain is activated. $\sigma_{j}(t)(j \in 1 \sim 3)$ is the adjustable weight, and $w_{h}(t)(h \in 1 \sim 3)$ is the Wiener process motion satisfying $E\left[d w_{h}(t)\right]=0$ and $E\left[\left(d w_{h}(t)\right)^{2}\right\rfloor=d t$. The synchronization error is defined by $e_{x}(t)=x_{m}(t)-x_{s}(t), e_{y}(t)=$ $y_{m}(t)-y_{s}(t)$, and $e_{z}(t)=z_{m}(t)-z_{s}(t)$.

Define an indicator function that $\xi(t)=\left(\xi_{1}(t), \xi_{2}(t), \ldots, \xi_{N}(t)\right)^{T}$ with

(i) if $0 \leq t<T$, then

$$
\xi_{i}(t)= \begin{cases}1, & \text { when the } i \text { th mode actives at time } \frac{T}{N}(i-1) \leq t<\frac{T}{N} i \\ 0, & \text { otherwise, }\end{cases}
$$

(ii) if $T \leq t$, then

$$
\xi_{i}(t+v T)=\xi_{i}(t), \quad v=1,2,3, \ldots,
$$

where $i=1,2,3, \ldots, N$ and $T$ is a period of time. 
Then, the master and salve systems can be respectively rewritten as

$$
\begin{gathered}
d x_{m}(t)=\sum_{i=1}^{N} \xi_{i}(t) \kappa_{i}\left\{\left[p\left(y_{m}(t)-x_{m}(t)-f\left(x_{m}(t)\right)\right)\right] d t\right\}, \\
d y_{m}(t)=\sum_{i=1}^{N} \xi_{i}(t) \kappa_{i}\left\{\left[x_{m}(t)-y_{m}(t)+z_{m}(t)\right] d t\right\}, \\
d z_{m}(t)=\sum_{i=1}^{N} \xi_{i}(t) \kappa_{i}\left\{\left[-q y_{m}(t)-r z_{m}(t)\right] d t\right\}, \\
d x_{s}(t)=\sum_{i=1}^{N} \xi_{i}(t) \kappa_{i}\left\{\left[p\left(y_{s}(t)-x_{s}(t)-f\left(x_{s}(t)\right)\right)\right] d t+\sigma_{1}(t) e_{x}(t) d w_{1}(t)\right\}, \\
d y_{s}(t)=\sum_{i=1}^{N} \xi_{i}(t) \kappa_{i}\left\{\left[x_{s}(t)-y_{s}(t)+z_{s}(t)-u(t)\right] d t+\sigma_{2}(t) e_{y}(t) d w_{2}(t)\right\}, \\
d z_{s}(t)=\sum_{i=1}^{N} \xi_{i}(t) \kappa_{i}\left\{\left[-q y_{s}(t)-r z_{s}(t)\right] d t+\sigma_{3}(t) e_{z}(t) d w_{3}(t)\right\} .
\end{gathered}
$$

It is noted that $\sum_{i=1}^{N} \xi_{i}(t)=1$ under all switching rules.

Then, the dynamics of synchronization error between the master and slave systems, (2.5) can be described by

$$
\begin{gathered}
d e_{x}(t)=\sum_{i=1}^{N} \xi_{i}(t) \kappa_{i}\left\{\left[p\left(e_{y}(t)-e_{x}(t)-f\left(e_{x}(t)\right)\right)\right] d t-\sigma_{1}(t) e_{x}(t) d w_{1}(t)\right\}, \\
d e_{y}(t)=\sum_{i=1}^{N} \xi_{i}(t) \kappa_{i}\left\{\left[e_{x}(t)-e_{y}(t)+e_{z}(t)+u(t)\right] d t-\sigma_{2}(t) e_{y}(t) d w_{2}(t)\right\}, \\
d e_{z}(t)=\sum_{i=1}^{N} \xi_{i}(t) \kappa_{i}\left\{\left[-q e_{y}(t)-z e_{z}(t)\right] d t-\sigma_{3}(t) e_{z}(t) d w_{3}(t)\right\},
\end{gathered}
$$

where $f\left(e_{x}(t)\right)=f\left(x_{m}(t)\right)-f\left(x_{s}(t)\right)$.

The main objective of control development in this paper is to select an appropriate switching surface and to design a sliding-mode controller to guarantee partial finite-time synchronization between the master and slave switched stochastic Chua's circuit systems. The first step is to select an appropriate switching surface to ensure the stochastic stability of 
the sliding motion on the sliding manifold.

$$
\begin{aligned}
s(t)= & e_{y}(t)+\psi(t), \\
\dot{\psi}(t)= & -\frac{\beta_{2} e_{y}^{2}(t)}{|s(t)|} \operatorname{sgn}(s(t))-\left(\eta_{1}+\eta_{2}|s(t)|^{\alpha}\right) \operatorname{sgn}(s(t))+\left(\eta_{3}+\eta_{4}\left|e_{y}(t)\right|^{\alpha}\right) \operatorname{sgn}\left(e_{y}(t)\right) \\
& +p e_{x}(t)+\beta_{2} e_{y}(t)+\left(\frac{\beta_{1} e_{x}^{2}(t)+p \ell\left|e_{x}(t)\right|^{2}+\left(\eta_{3}\left|e_{x}(t)\right|+\eta_{4}\left|e_{x}(t)\right|^{\alpha+1}\right)}{\left|e_{y}(t)\right|}\right) \operatorname{sgn}\left(e_{y}(t)\right),
\end{aligned}
$$

where $s \in R^{1}, \beta_{1}$, and $\beta_{2}$ are two setting positive constant such that $\beta_{1}>(1 / 2) \sigma_{1}^{2}(t)$ and $\beta_{2}>$ $(1 / 2) \sigma_{2}^{2}(t)$, respectively. $\eta_{i}(i \in 1 \sim 4)$ are the sliding-mode controller gains which are positive constants. When system (2.6a)-(2.6c) is in the sliding mode, the condition $E[s(t)]=E[\dot{s}(t)]=$ 0 has to be satisfied. Then, the stochastic process of the sliding surface $s(t)$ is considered as follows.

The time integration of the error dynamic equations $\dot{e}_{y}(t)$ is

$$
e_{y}(t)=\sum_{i=1}^{N} \xi_{i}(t) \kappa_{i}\left\{e_{y}(0)+\int_{0}^{t}\left[e_{x}(\tau)-e_{y}(\tau)+e_{z}(\tau)+u(\tau)\right] d \tau-\sigma_{2}(\tau) e_{y}(\tau) d w_{2}(\tau)\right\} .
$$

Combining (2.7) and (2.9) yields

$$
s(t)=\sum_{i=1}^{N} \xi_{i}(t) \kappa_{i}\left\{e_{y}(0)+\psi(t)+\int_{0}^{t}\left(\left(e_{x}(\tau)-e_{y}(\tau)+e_{z}(\tau)+u_{1}(\tau)\right) d \tau-\sigma_{2}(\tau) e_{y}(\tau) d w_{2}(\tau)\right)\right\}
$$

From (2.10), we can obtain the following:

$$
d s(t)=\sum_{i=1}^{N} \xi_{i}(t) \kappa_{i}\left\{\left(e_{x}(t)-e_{y}(t)+e_{z}(t)+u(t)+\dot{\psi}(t)\right) d t-\sigma_{2}(t) e_{y}(t) d w_{2}(t)\right\}
$$

In order to derive the main results, the following lemma is needed.

Lemma 2.1 (see [30]). Assume that a continuous, positive-definite function $V(t)$ satisfies the following differential inequality:

$$
\dot{V}(t) \leq-\Delta V^{\alpha}(t), \quad \forall t \geq t_{0}, V\left(t_{0}\right) \geq 0,
$$

where $\Delta>0$ and $0<\alpha<1$ are two constants. Then, for any given $t_{0}, V(t)$ satisfies the following inequality:

$$
V^{1-\alpha}(t) \leq V^{1-\alpha}\left(t_{0}\right)-\Delta(1-\alpha)\left(t-t_{0}\right), \quad t_{0} \leq t \leq t_{r}
$$


$V(t) \equiv 0$, for all $t \geq t_{r}$, with $t_{r}$ given by

$$
t_{r}=t_{0}+\frac{V^{1-\alpha}(t)}{\Delta(1-\alpha)}
$$

According to the Lyapunov stability theorem and Lemma 2.1, if there is a sliding-mode controller such that $V_{s} \leq-\Delta V_{s}^{\alpha}(t)$, where $V_{s}=(1 / 2) s^{2}(t)$ is the defined Lyapunov function, and $\Delta>0$ and $0<\alpha<1$ are two real constants, the error dynamics converging to the sliding surface and $E[s(t)]=0$ reaching in finite time can be achieved. Therefore, the second step is to design the proposed sliding-mode controller $u(t)$ which is

$$
\begin{aligned}
u(t)= & -(1+p) e_{x}(t)+e_{y}(t)-e_{z}(t)-\beta_{2} e_{y}(t)-\left(\eta_{3}+\eta_{4}\left|e_{y}(t)\right|^{\alpha}\right) \operatorname{sgn}\left(e_{y}(t)\right) \\
& -\left(\frac{\beta_{1} e_{x}^{2}(t)+p \ell\left|e_{x}(t)\right|^{2}+\left(\eta_{3}\left|e_{x}(t)\right|+\eta_{4}\left|e_{x}(t)\right|^{\alpha+1}\right)}{\left|e_{y}(t)\right|}\right) \operatorname{sgn}\left(e_{y}(t)\right) .
\end{aligned}
$$

Theorem 2.2. By setting the sliding-mode controller in (2.15), the error dynamics in (2.6a)-(2.6c) will converge to the sliding surface, and $E[s(t)]=0$ is reached in finite time.

Proof. Define Lyapunov function

$$
V_{s}(t)=\frac{1}{2} s^{2}(t)=\frac{1}{2}|s(t)|^{2}
$$

By using the Ito formula, one can obtain that

$$
\begin{aligned}
E\left[V_{s}(t)\right]=E\left[\sum_{i=1}^{N} \xi_{i}(t) \kappa_{i}\{s(\right. & e_{x}(t)-e_{y}(t)+e_{z}(t)-(1+p) e_{x}(t) \\
& +e_{y}(t)-e_{z}(t)-\beta_{2} e_{y}(t)-\left(\eta_{3}+\eta_{4}\left|e_{y}(t)\right|^{\alpha}\right) \operatorname{sgn}\left(e_{y}(t)\right) \\
& -\left(\frac{\beta_{1} e_{x}^{2}(t)+p \ell\left|e_{x}(t)\right|^{2}+\left(\eta_{3}\left|e_{x}(t)\right|+\eta_{4}\left|e_{x}(t)\right|^{\alpha+1}\right)}{\left|e_{y}(t)\right|}\right) \operatorname{sgn}\left(e_{y}(t)\right) \\
& +p e_{x}(t)+\beta_{2} e_{y}(t)-\frac{\beta_{2} e_{y}^{2}(t)}{|s(t)|} \operatorname{sgn}(s(t))-\left(\eta_{1}+\eta_{2}|s(t)|^{\alpha}\right) \operatorname{sgn}(s(t)) \\
& +\left(\frac{\beta_{1} e_{x}^{2}(t)+p \ell\left|e_{x}(t)\right|^{2}+\left(\eta_{3}\left|e_{x}(t)\right|+\eta_{4}\left|e_{x}(t)\right|^{\alpha+1}\right)}{\left|e_{y}(t)\right|}\right) \operatorname{sgn}\left(e_{y}(t)\right) \\
& \left.\left.\left.+\left(\eta_{3}+\eta_{4}\left|e_{y}(t)\right|^{\alpha}\right) \operatorname{sgn}\left(e_{y}(t)\right)\right)+\frac{1}{2}\left(\sigma_{2}(t) e_{y}(t)\right)^{2}\right\}\right]
\end{aligned}
$$


Since $\beta_{2}>(1 / 2) \sigma_{2}^{2}(t)$, regarding the above inequality,

$$
\begin{aligned}
E\left[V_{s}(t)\right] & \leq E\left[\sum_{i=1}^{N} \xi_{i}(t) \kappa_{i}\left\{s\left(-\left(\eta_{1}+\eta_{2}|s(t)|^{\alpha}\right) \operatorname{sgn}(s(t))\right)\right\}\right] \\
& \leq E\left[\sum_{i=1}^{N} \xi_{i}(t) \kappa_{i}\left\{-\eta_{1}|s(t)|-\eta_{2}|s(t)|^{\alpha+1}\right\}\right] \\
& \leq E\left[\sum_{i=1}^{N} \xi_{i}(t) \kappa_{i}\left\{-2^{(\alpha+1) / 2} \eta_{2} V_{s}(t)^{(\alpha+1) / 2}\right\}\right] .
\end{aligned}
$$

From Lemma 2.1, it implies that $E[s(t)]=0$ in finite time with the controller in (2.15), completing the proof.

Theorem 2.3. Based on the design-switching surface in (2.7) and the controller in (2.15), the partial finite-time synchronization of the sliding motion on the sliding manifold is guaranteed. Then, the mean of $E[e(t)]$ on the sliding manifold can achieve the partial finite-time stability.

Proof. Define Lyapunov function

$$
V_{e}(t)=\frac{1}{2} e_{x}^{2}(t)+\frac{1}{2} e_{y}^{2}(t)
$$

By using the Ito formula, one can obtain that

$$
\begin{aligned}
E\left[\dot{V}_{e}(t)\right]=E\left[\sum_{i=1}^{N} \xi_{i}(t) \kappa_{i}\{\right. & e_{x}(t)\left(-p e_{x}(t)+p e_{y}(t)-p f\left(e_{x}(t)\right)\right)+\frac{1}{2} \sigma_{1}^{2} e_{x}^{2}(t) \\
& +e_{y}(t)\left(e_{x}(t)-e_{y}(t)+e_{z}(t)-(1+p) e_{x}(t)+e_{y}(t)-e_{z}(t)-\beta_{2} e_{y}(t)\right. \\
& -\left(\frac{\beta_{1} e_{x}^{2}(t)+p \ell\left|e_{x}(t)\right|^{2}+\left(\eta_{3}\left|e_{x}(t)\right|+\eta_{4}\left|e_{x}(t)\right|^{\alpha+1}\right)}{\left|e_{y}(t)\right|}\right) \operatorname{sgn}\left(e_{y}(t)\right) \\
& \left.\left.\left.-\left(\eta_{3}+\eta_{4}\left|e_{y}(t)\right|^{\alpha}\right) \operatorname{sgn}\left(e_{y}(t)\right)\right)+\frac{1}{2} \sigma_{2}^{2}(t) e_{y}^{2}(t)\right\}\right]
\end{aligned}
$$


Based on the Lipschitz condition and setting $\beta_{1}>(1 / 2) \sigma_{1}^{2}(t)$ and $\beta_{2}>(1 / 2) \sigma_{2}^{2}(t)$, regarding the above inequality,

$$
\begin{aligned}
E\left[\dot{V}_{e}(t)\right] & \leq E\left\lfloor\sum_{i=1}^{N} \xi_{i}(t) \kappa_{i}\left\{-\eta_{3}\left|e_{x}(t)\right|-\eta_{4}\left|e_{x}(t)\right|^{\alpha+1}-\eta_{3}\left|e_{y}(t)\right|-\eta_{4}\left|e_{y}(t)\right|^{\alpha+1}\right\}\right\rfloor \\
& \leq E\left\lfloor\sum_{i=1}^{N} \xi_{i}(t) \kappa_{i}\left\{-\eta_{4}\left|e_{x}(t)\right|^{\alpha+1}-\eta_{4}\left|e_{y}(t)\right|^{\alpha+1}\right\}\right\rfloor .
\end{aligned}
$$

From the above equation, we can obtain that

$$
\begin{aligned}
& E\left[\dot{V}_{e}(t)\right] \leq E\left\lfloor\sum_{i=1}^{N} \xi_{i}(t) \kappa_{i}\left\{-\eta_{4}\left|e_{x}(t)\right|^{\alpha+1}\right\}\right], \\
& E\left[\dot{V}_{e}(t)\right] \leq E\left\lfloor\sum_{i=1}^{N} \xi_{i}(t) \kappa_{i}\left\{-\eta_{4}\left|e_{x}(t)\right|^{\alpha+1}\right\}\right\rfloor .
\end{aligned}
$$

Then, they can be rewritten as

$$
\begin{aligned}
& E\left[\dot{V}_{e}^{2 /(\alpha+1)}(t)\right] \leq E\left[\sum_{i=1}^{N} \xi_{i}(t) \kappa_{i}\left\{-2 \eta_{4}^{2 /(\alpha+1)}\left(\frac{1}{2}\left|e_{x}(t)\right|^{2}\right)\right\}\right], \\
& E\left[\dot{V}_{e}^{2 /(\alpha+1)}(t)\right] \leq E\left[\sum_{i=1}^{N} \xi_{i}(t) \kappa_{i}\left\{-2 \eta_{4}^{2 /(\alpha+1)}\left(\frac{1}{2}\left|e_{y}(t)\right|^{2}\right)\right\}\right] .
\end{aligned}
$$

It implies that

$$
\begin{gathered}
E\left[2 \dot{V}_{e}^{2 /(\alpha+1)}(t)\right] \leq E\left[\sum_{i=1}^{N} \xi_{i}(t) \kappa_{i}\left\{-2 \eta_{4}^{2 /(\alpha+1)}\left(\frac{1}{2}\left|e_{x}(t)\right|^{2}\right)-2 \eta_{4}^{2 /(\alpha+1)}\left(\frac{1}{2}\left|e_{y}(t)\right|^{2}\right)\right\}\right] \\
E\left[\dot{V}_{e}^{2 /(\alpha+1)}(t)\right] \leq E\left[\sum_{i=1}^{N} \xi_{i}(t) \kappa_{i}\left\{-\eta_{4}^{2 /(\alpha+1)}\left(\frac{1}{2}\left(\left|e_{x}(t)\right|^{2}+\left|e_{y}(t)\right|^{2}\right)\right)\right\}\right]
\end{gathered}
$$

Therefore, we can get that

$$
\begin{aligned}
E\left[\dot{V}_{e}(t)\right] & \leq E\left[\sum_{i=1}^{N} \xi_{i}(t) \kappa_{i}\left\{-\eta_{4}\left(\frac{1}{2}\left(\left|e_{x}(t)\right|^{2}+\left|e_{y}(t)\right|^{2}\right)\right)^{(\alpha+1) / 2}\right\}\right] \\
& \leq E\left[\sum_{i=1}^{N} \xi_{i}(t) \kappa_{i}\left\{-\eta_{4}\left(V_{e}\right)^{(\alpha+1) / 2}\right\}\right] .
\end{aligned}
$$

From Lemma 2.1, $E\left[e_{x}(t)\right]$ and $E\left[e_{y}(t)\right]$ can converge to zero in finite time $t_{r}$ along the sliding surface. Then, from the error dynamic (2.6c), $E\left[e_{z}(t)\right]$ can tend to zero as $E\left[e_{y}(t)\right]$ converging 


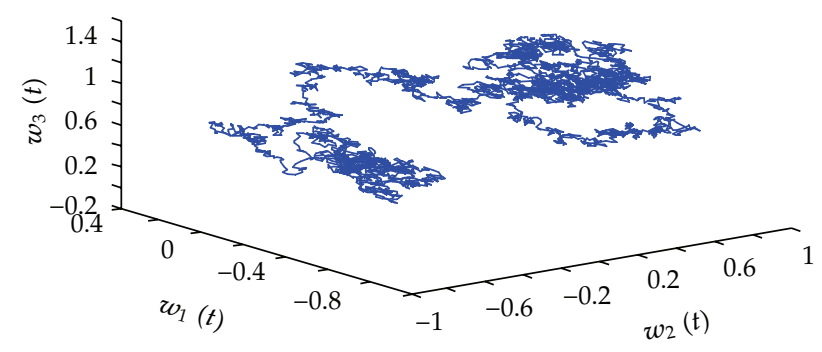

Figure 1: Time responses of the Wiener process motion $w_{h}(t)$.

to zero in finite-time $t_{r}$. It implies that the asymptotical stability of $E\left[e_{z}(t)\right]$ can be achieved after the time $t_{r}$. Based on the above proof, the partial finite-time synchronization of the sliding motion on the sliding manifold is guaranteed, completing the proof.

Remark 2.4. From the system (2.1), $f(x(t))=b x(t)+(1 / 2)(a-b)[|x(t)+1|-|x(t)-1|]$ can be rewritten as

$$
f(x(t))= \begin{cases}b x(t)+a-b, & \text { if } x(t)>1 \\ a x(t), & \text { if }|x(t)| \leq 1 \\ b x(t)-a+b, & \text { if } x(t)<-1\end{cases}
$$

Therefore, we can have

$$
\left|f\left(x_{m}(t)\right)-f\left(x_{s}(t)\right)\right|= \begin{cases}\left|b x_{m}(t)-b x_{s}(t)\right|, & \text { if } x(t)>1 \\ \left|a x_{m}(t)-a x_{s}(t)\right|, & \text { if }|x(t)| \leq 1 \\ \left|b x_{m}(t)-b x_{s}(t)\right|, & \text { if } x(t)<-1\end{cases}
$$

From the definition of the Chua's circuit [31], $|a|>|b|$ can be obtained. It implies that the following inequality is achieved:

$$
\left|f\left(x_{m}(t)\right)-f\left(x_{s}(t)\right)\right| \leq|a|\left|x_{m}(t)-x_{s}(t)\right|
$$

From the above reasoning, it can be sure that $f(x(t))$ satisfies the Lipschitz condition with Lipschitz constant $\ell \geq|a|$.

Remark 2.5. In order to avoid chattering, $\operatorname{sgn}(s(t))$ is replaced with $s(t) /(|s(t)|+\wp)$ in the simulation, where $\wp$ is an appropriate minimal value.

\section{An Illustrative Example}

Consider the proposed synchronization of switched stochastic Chua's circuits with the parameters given by $a=-1.28, b=-0.69, p=10, q=15, r=0.0385$, and $T=50$ (sec). The 


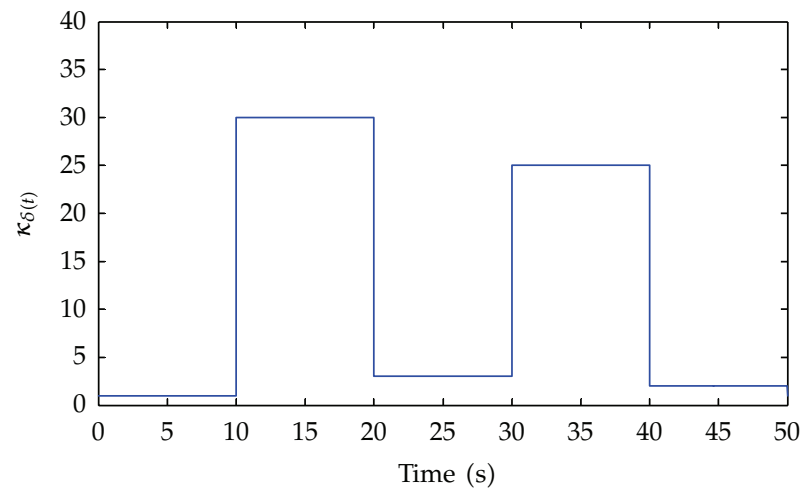

Figure 2: Time responses of piecewise switching signal $\kappa_{\delta(t)}$.
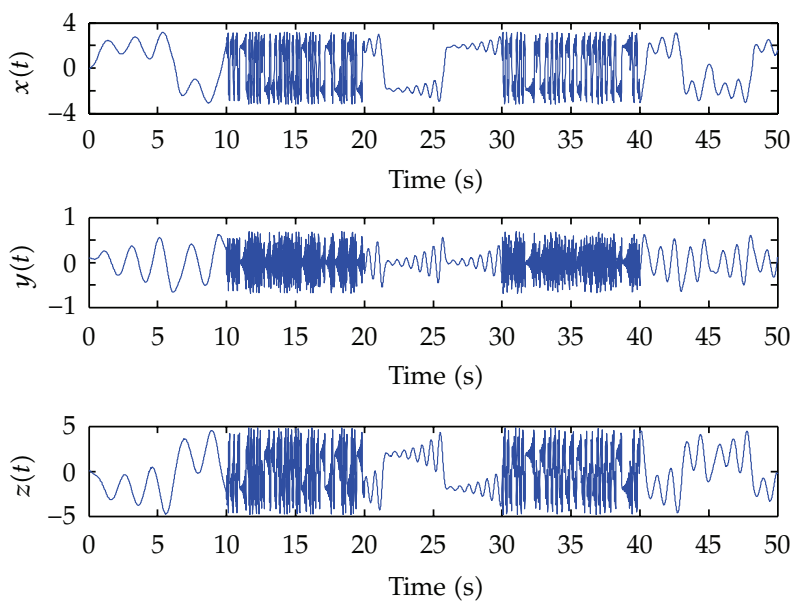

Figure 3: State responses of the switched stochastic Chua's system.
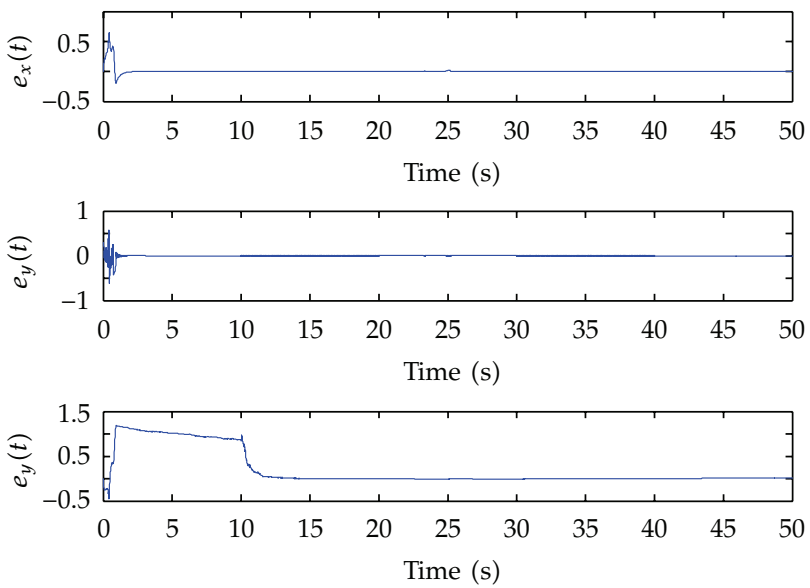

Figure 4: Time responses of the synchronization error. 

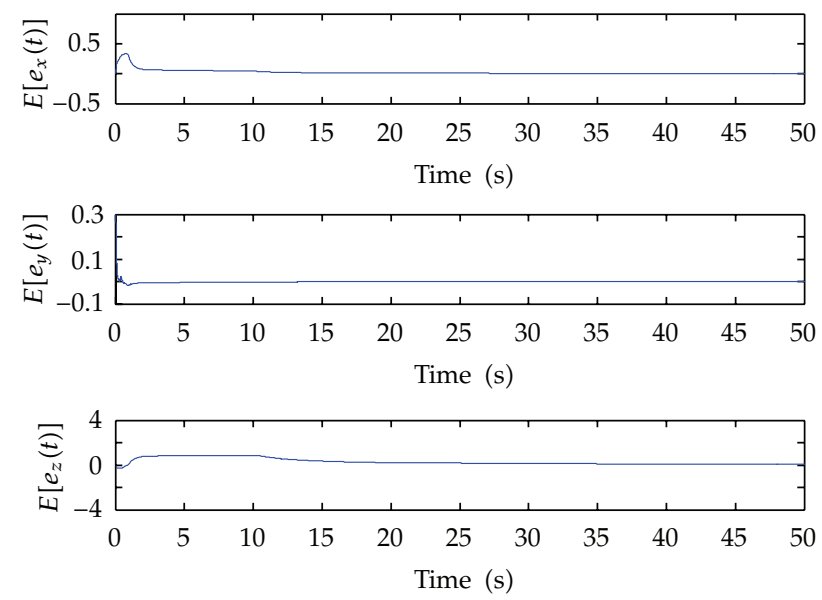

Figure 5: Time responses of the mean values of the synchronization error.
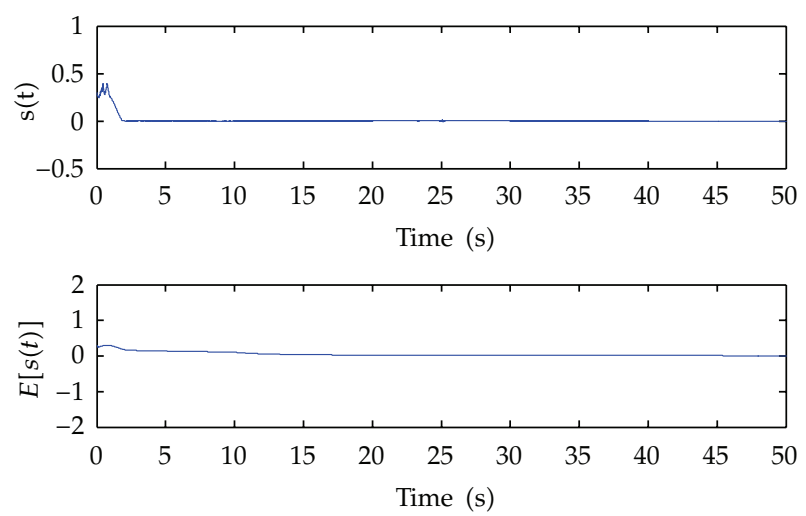

Figure 6: Time responses and mean values of the sliding surface function $s(t)$.

parameters for the sliding surface and sliding mode controller are given by $\alpha=0.9, \beta_{1}=\beta_{2}=$ $1, \ell=1.3$, and the sliding-mode controller gains are given by $\eta_{1}=\eta_{2}=\eta_{3}=\eta_{4}=0.3$. $w_{h}(t)$ is the Wiener process motion with time $T$, shown in Figure 1 , and $\sigma_{1}(t)=\sigma_{2}(t)=\sigma_{3}(t)=0.1$. The real constant $\wp=10^{-3}$ is given. Time response of the piecewise switching signal $\kappa_{\delta(t)}$ is shown in Figure 2. With the modulation of the time-driven switching rule, the state responses behave like frequency modulation. The state responses of the stochastic switched Chua's circuits are shown in Figure 3, and the speed of response is different with different system gains. Based on the proposed controller, the partial finite-time stability of the sliding motion on the sliding manifold is shown in Figure 4 which displays the synchronization errors of the stochastic switched Chua's circuits. The mean values of synchronization errors on the sliding manifold reaching the partial finite-time stability are shown in Figure 5. In Figure 6, time responses and mean value of the sliding surface function $s(t)$ are shown, and it also reveals that $E[s(t)]=0$ is reached in finite time. According to the above simulation, partial finite time synchronization between switched stochastic Chua's circuits and the mean value of the error states reaching zero in finite time on the sliding manifold are guaranteed by the proposed controller. 


\section{Conclusion}

This study investigated the partial finite-time synchronization problem of stochastic Chua's circuits with switched gains which depend on a time-driven switching law. Based on the Ito formula and Lyapunov stability theory, a sliding-mode controller was proposed to synchronize the switching master and slave stochastic Chua's circuits. The mean value of the error states reaching zero in finite time was demonstrated. Numerical simulations show the effectiveness of the proposed method.

\section{Acknowledgment}

This work was supported by the National Science Council of Republic of China under contract 100-2221-E-366-001 and 100-2632-E-366-001-MY3.

\section{References}

[1] C.-H. Kao, C.-F. Hsu, C.-H. Wang, and H.-S. Don, “Chaos synchronization using adaptive dynamic neural network controller with variable learning rates," Mathematical Problems in Engineering, vol. 2011, Article ID 701671, 20 pages, 2011.

[2] T. L. Liao and S. H. Tsai, "Adaptive synchronization of chaotic systems and its application to secure communications," Chaos, Solitons and Fractals, vol. 11, no. 9, pp. 1387-1396, 2000.

[3] A. E. Matouk, "Chaos synchronization between two different fractional systems of Lorenz family," Mathematical Problems in Engineering, vol. 2009, Article ID 572724, 11 pages, 2009.

[4] Y. Y. Hou, T. L. Liao, and J. J. Yan, " $H_{\infty}$ synchronization of chaotic systems using output feedback control design," Physica A, vol. 379, no. 1, pp. 81-89, 2007.

[5] J.-H. Kim, C.-W. Park, E. Kim, and M. Park, "Adaptive synchronization of T-S fuzzy chaotic systems with unknown parameters," Chaos, Solitons and Fractals, vol. 24, no. 5, pp. 1353-1361, 2005.

[6] D. Liberzon, Switching in Systems and Control, Systems \& Control: Foundations \& Applications, Birkhäuser, Boston, Mass, USA, 2003.

[7] J. Daafouz, P. Riedinger, and C. Iung, "Stability analysis and control synthesis for switched systems: a switched Lyapunov function approach," IEEE Transactions on Automatic Control, vol. 47, no. 11, pp. 1883-1887, 2002.

[8] X.-M. Sun, W. Wang, G.-P. Liu, and J. Zhao, "Stability analysis for linear switched systems with timevarying delay," IEEE Transactions on Systems, Man, and Cybernetics, Part B, vol. 38, no. 2, pp. 528-533, 2008.

[9] V. F. Montagner, V. J. S. Leite, R. C. L. F. Oliveira, and P. L. D. Peres, "State feedback control of switched linear systems: an LMI approach," Journal of Computational and Applied Mathematics, vol. 194, no. 2, pp. 192-206, 2006.

[10] H. Lin and P. J. Antsaklis, "Stability and stabilizability of switched linear systems: a survey of recent results," IEEE Transactions on Automatic Control, vol. 54, no. 2, pp. 308-322, 2009.

[11] P. Colaneri, J. C. Geromel, and A. Astolfi, "Stabilization of continuous-time switched nonlinear systems," Systems \& Control Letters, vol. 57, no. 1, pp. 95-103, 2008.

[12] Z. Wang, H. Shu, and X. Liu, "Reliable stabilization of stochastic time-delay systems with nonlinear disturbances," International Journal of General Systems, vol. 34, no. 5, pp. 523-535, 2005.

[13] S. Ma and E. K. Boukas, "Stability and robust stabilisation for uncertain discrete stochastic hybrid singular systems with time delay," IET Control Theory \& Applications, vol. 3, no. 9, pp. 1217-1225, 2009.

[14] K. Benjelloun and E. K. Boukas, "Mean square stochastic stability of linear time-delay system with Markovian jumping parameters," IEEE Transactions on Automatic Control, vol. 43, no. 10, pp. 14561460, 1998.

[15] Q.-C. Pham, N. Tabareau, and J.-J. Slotine, "A contraction theory approach to stochastic incremental stability," IEEE Transactions on Automatic Control, vol. 54, no. 4, pp. 816-820, 2009.

[16] A. Hu and $\mathrm{Z}$. Xu, "Stochastic linear generalized synchronization of chaotic systems via robust control," Physics Letters A, vol. 372, no. 21, pp. 3814-3818, 2008. 
[17] Y. Sun and J. Cao, "Adaptive lag synchronization of unknown chaotic delayed neural networks with noise perturbation," Physics Letters A, vol. 364, no. 3-4, pp. 277-285, 2007.

[18] H. Salarieh and A. Alasty, "Adaptive synchronization of two chaotic systems with stochastic unknown parameters," Communications in Nonlinear Science and Numerical Simulation, vol. 14, no. 2, pp. 508-519, 2009.

[19] W. Yu and J. Cao, "Synchronization control of stochastic delayed neural networks," Physica A, vol. 373, pp. 252-260, 2007.

[20] J.-J. Yan, Y.-S. Yang, T.-Y. Chiang, and C.-Y. Chen, "Robust synchronization of unified chaotic systems via sliding mode control," Chaos, Solitons and Fractals, vol. 34, no. 3, pp. 947-954, 2007.

[21] L. Boutat-Baddas, J. P. Barbot, D. Boutat, and R. Tauleigne, "Sliding mode observers and observability singularity in chaotic synchronization," Mathematical Problems in Engineering, vol. 2004, no. 1, pp. 1131,2004

[22] D. Chen and W. Zhang, "Sliding mode control of uncertain neutral stochastic systems with multiple delays," Mathematical Problems in Engineering, vol. 2008, Article ID 761342, 9 pages, 2008.

[23] H.-T. Yau, "Chaos synchronization of two uncertain chaotic nonlinear gyros using fuzzy sliding mode control," Mechanical Systems and Signal Processing, vol. 22, no. 2, pp. 408-418, 2008.

[24] S. H. Hosseinnia, R. Ghaderi, A. Ranjbar N., M. Mahmoudian, and S. Momani, "Sliding mode synchronization of an uncertain fractional order chaotic system," Computers $\mathcal{E}$ Mathematics with Applications, vol. 59, no. 5, pp. 1637-1643, 2010.

[25] H. Wang, Z.-Z. Han, Q.-Y. Xie, and W. Zhang, "Finite-time chaos control via nonsingular terminal sliding mode control," Communications in Nonlinear Science and Numerical Simulation, vol. 14, no. 6, pp. 2728-2733, 2009.

[26] E. Moulay and W. Perruquetti, "Finite time stability and stabilization of a class of continuous systems," Journal of Mathematical Analysis and Applications, vol. 323, no. 2, pp. 1430-1443, 2006.

[27] X. Huang, W. Lin, and B. Yang, "Global finite-time stabilization of a class of uncertain nonlinear systems," Automatica, vol. 41, no. 5, pp. 881-888, 2005.

[28] S. G. Nersesov and W. M. Haddad, "Finite-time stabilization of nonlinear impulsive dynamical systems," Nonlinear Analysis: Hybrid Systems, vol. 2, no. 3, pp. 832-845, 2008.

[29] C. Jammazi, "On a sufficient condition for finite-time partial stability and stabilization: applications," IMA Journal of Mathematical Control and Information, vol. 27, no. 1, pp. 29-56, 2010.

[30] E. Moulay, M. Dambrine, N. Yeganefar, and W. Perruquetti, "Finite-time stability and stabilization of time-delay systems," Systems \& Control Letters, vol. 57, no. 7, pp. 561-566, 2008.

[31] W. Li and X. Chen, "Projective synchronization of Chua's chaotic system with dead-zone in the control input," Communications in Nonlinear Science and Numerical Simulation, vol. 14, no. 7, pp. 3100-3107, 2009. 


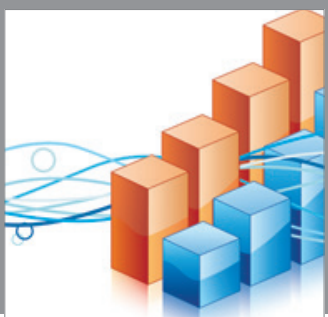

Advances in

Operations Research

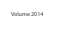

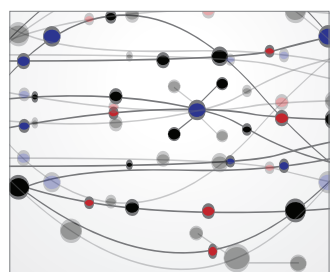

\section{The Scientific} World Journal
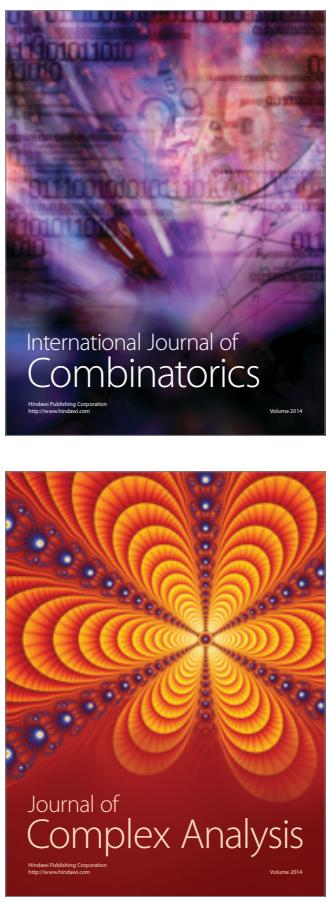

International Journal of

Mathematics and

Mathematical

Sciences
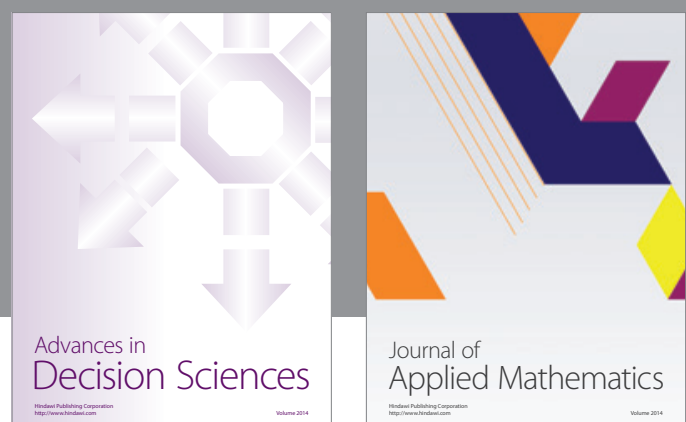

Journal of

Applied Mathematics
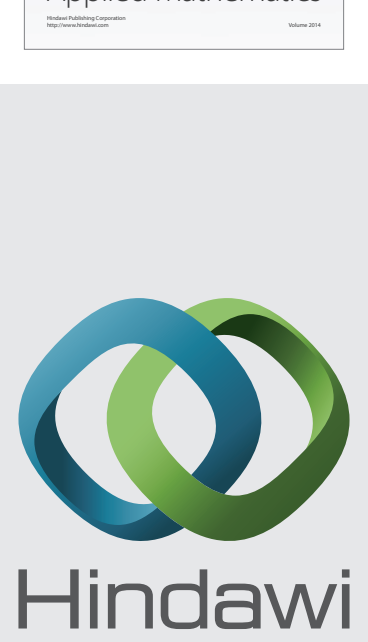

Submit your manuscripts at http://www.hindawi.com
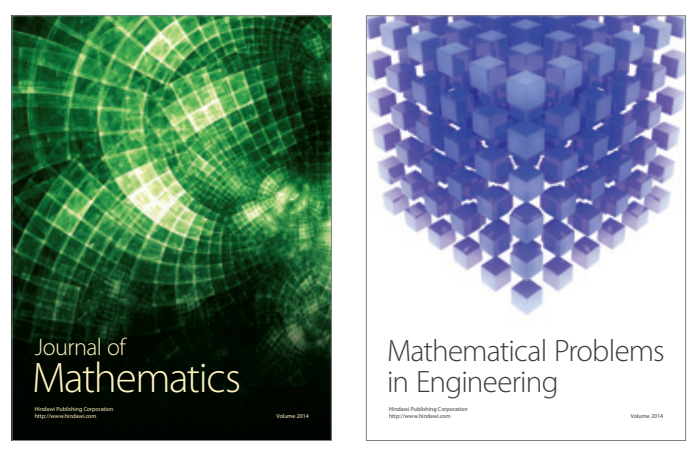

Mathematical Problems in Engineering
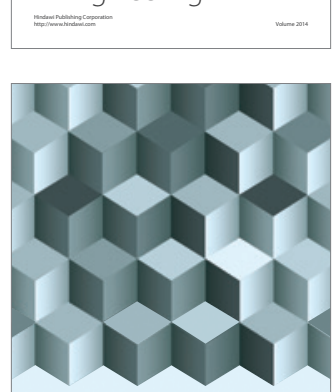

Journal of

Function Spaces
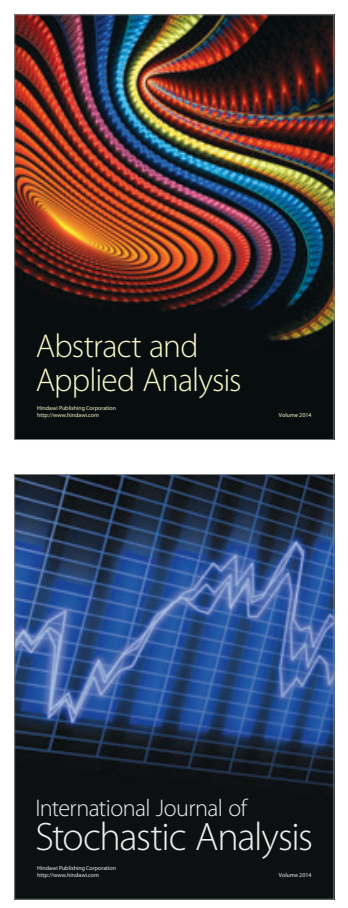

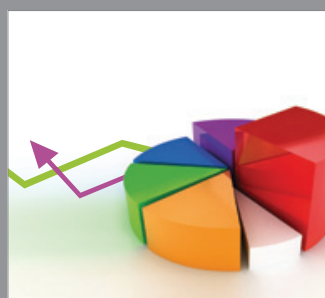

ournal of

Probability and Statistics

Promensencen
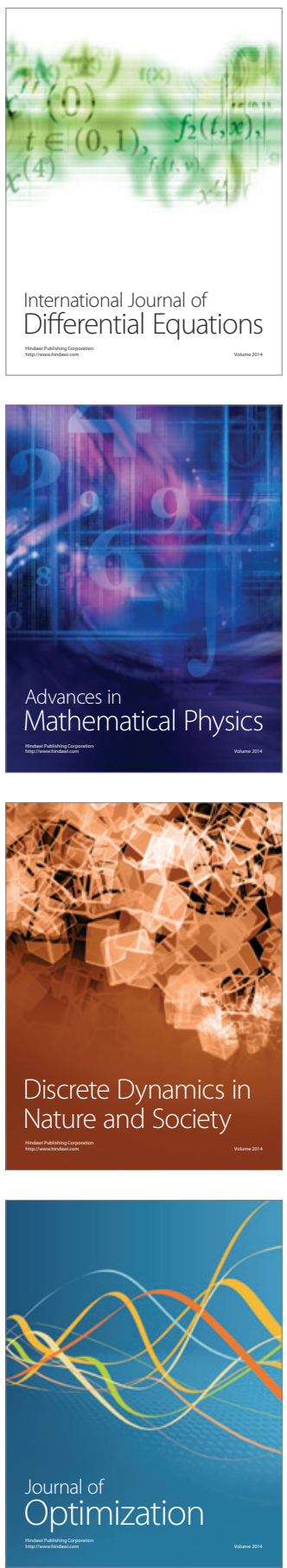